\title{
A new method for parameterization of phase shift and backscattering amplitude
}

\author{
Marius Vaarkamp ${ }^{\mathrm{a}, \mathrm{b}, *}$, Johannes C. Linders ${ }^{\mathrm{a}}$, Diek C. Koningsberger ${ }^{\mathrm{c}}$ \\ ${ }^{a}$ Eindhoven University of Technology, P.O. Box 513, $5600 \mathrm{MB}$ Eindhoven, The Netherlands \\ ${ }^{b} X A F S$ Services International, P.O. Box 24040, 3502 MA Utrecht, The Netherlands \\ ${ }^{'}$ Department of Inorganic Chemistry, Debye Institute, Utrecht University, P.O. Box 80083, 3508 TB, Utrecht, The Netherlands
}

\begin{abstract}
Parameterization of phase and backscattering amplitude with cubic splines is described. Using these cubic spline, the analytical partial derivatives of the plane wave EXAFS function can be calculated. The use of analytical partial derivatives decreases the CPU time needed for a refinement by over $60 \%$ for a three shell system compared to a refinement with partial derivatives calculated with the finite difference method.
\end{abstract}

\section{Introduction}

XAS experiments are in principle suited to yield detailed information on the local structure around the absorbing atom. The information is however buried in complicated mathematical formulas. The most widely used method to extract the information from the experimental data is the comparison of the data with a model spectrum. By adjusting the structural parameters the resemblance of data and model spectrum is improved. The direction and magnitude of the adjustments to the structural parameters are generally calculated using non-linear least squares methods.

Due to the discontinuity of the phase and backscattering amplitude function the partial derivatives, required for non-linear least squares methods, can only be calculated using the finite difference method. The parameterization with cubic splines, presented here, makes these functions continuous. The now available analytical

\footnotetext{
${ }^{*}$ Corresponding author.
}

partial derivatives reduce the $\mathrm{CPU}$ time required for refinement of the structural parameters.

\section{Description, implementation and discussion}

An accurate theoretical description of EXAFS spectra includes curved wave effects and an energy dependent self-energy [1]. At every distance the curved wave EXAFS formula reduces to the plane-wave EXAFS formula (1), which is the model function used in most refinement procedures:

$$
\begin{aligned}
\chi(k)=\sum_{j=1}^{\text {shells }} & S_{0}^{2} \frac{N_{j}}{k R_{j}^{2}} \mathrm{e}^{\left(-2 R_{j} / \lambda\right)} \mathrm{e}^{-2 k^{2} \sigma_{j}^{2}} \\
& \times F_{j}(k) \sin \left(2 k R_{j}+\varphi_{j}(k)\right) .
\end{aligned}
$$

Besides the coordination number $N$, the distance $R$ and the mean square relative displacement (DebyeWaller factor) $\sigma^{2}$, the quantities $S_{0}^{2}, \mathrm{e}^{(-2 R / \lambda)}, F(k)$, and $\phi(k)$ are unknown. The backscattering amplitude $F(k)$ and the phase $\phi(k)$ of an absorber-backscatterer pair can be extracted by Fourier filtering from a compound in 
which the contributions are well separated, or calculated from first principles. To calculate $F(k)$ from an inverse Fourier transform $S_{0}^{2}, \mathrm{e}^{(-2 R / \lambda)}$, and $\sigma^{2}$ have to be known. To circumvent this requirement it is assumed that apart from $F(k)$ and $\phi(k)$, also $S_{0}^{2}$ and $\mathrm{e}^{(-2 R / \lambda)}$ are transferable from one compound to another. The validity of this assumption has been shown for compounds with the same absorber-backscatterer pair [2,3], and even for absorbers or backscatterers which are neighbours in the periodic table $[4,5]$. This leads to the definition of a modified backscattering amplitude $\left(F^{\prime}(k)\right)$ :

$F^{\prime}(k)=S_{0}^{2} \mathrm{e}^{(-2 R / \lambda)} \mathrm{e}^{-2 k^{2} \sigma^{2}} F(k)$.

This modified backscattering amplitude contains the Debye-Waller factor of the reference compound. Hence, the Debye-Waller factor obtained during non-linear least squares refinement is relative to the Debye-Waller factor of the reference compound $\left(\Delta \sigma^{2}\right)$. The function to be minimized becomes

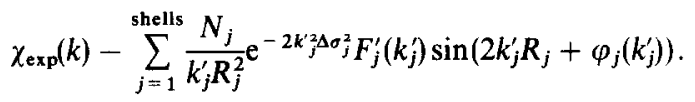

Here $k^{\prime}$ is the photo electron wave vector corrected for the difference in inner potential between the sample and the reference compound $\left(\Delta E_{0}\right)$ :

$k^{\prime}=\sqrt{k^{2}+\frac{2 m_{\mathrm{e}}}{\hbar^{2}} \Delta E_{0}}$

The structural parameters $N, R$, and $\Delta \sigma^{2}$ have to be refined for every contribution (shell).

Since $\Delta E_{0}$ is a fitting parameter, the values of $k^{\prime}$, and consequently $F^{\prime}\left(k^{\prime}\right)$ and $\varphi\left(k^{\prime}\right)$ are subject to change during a refinement. Hence, a fast and accurate method to calculate $F^{\prime}\left(k^{\prime}\right)$ and $\varphi\left(k^{\prime}\right)$ from the original $F^{\prime}(k)$ and $\phi(k)$ data is needed. The smooth nature of cubic splines makes them extremely well suited to describe the slowly varying phase and backscattering amplitude functions.

The cubic spline parameterization of phase and backscattering amplitude described has been implemented in XDAP. ${ }^{1}$ The time consuming calculation of the spline coefficients is carried out only once for each phase and backscattering amplitude function. Evaluation of a cubic spline and the derivative of a spline is fast due to a search algorithm that uses the knowledge that the points of the EXAFS model function are calculated with increasing energy values.

An advantage of the use of cubic splines is that the derivative of the amplitude and phase functions with

\footnotetext{
${ }^{1}$ XAFS Data Analysis Program developed by Eindhoven University of Technology, Utrecht University, and XAFS Services International.
}

Table 1

CPU time required for a refinement on a VAX 4200

\begin{tabular}{llcc}
\hline Shells & Free parameters & Numerical (s) & Analytical (s) \\
\hline 1 & 4 & 6.7 & 6.3 \\
2 & 4 & 10.6 & 8.2 \\
2 & 8 & 19.4 & 11.7 \\
3 & 4 & 8.3 & 13.9 \\
3 & 8 & 61.0 & 22.5 \\
\hline
\end{tabular}

respect to $\Delta E_{0}$ can be calculated. This offers the possibility to use analytical partial derivatives in the refinement procedure. Table 1 gives the CPU time needed for a refinement with the use of analytical partial derivatives and the numerical estimation of the partial derivatives with the finite difference method. The speed increase is $7 \%$ for a single shell system and increases to more than $60 \%$ for a three shell system.

The finite difference method calculates the complete spectrum for each partial derivative to be evaluated. To calculate an analytical partial derivative only the contribution of the parameter of which the partial derivative is evaluated has to be calculated. The reduction in the number of calculations required increases with the number of contributions to the model EXAFS spectrum. The reduction in CPU time for a single shell model EXAFS is due to the fact that all partial derivatives of a shell are calculated in a single pass, whereas in the finite difference method four EXAFS spectra with a single contribution have to be calculated.

\section{Conclusion}

Parameterization of phase and backscattering amplitude by cubic splines has been described. The analytical partial derivatives, calculated using the cubic splines, increase the speed of the refinement of structural parameters of a model EXAFS spectrum.

\section{References}

[1] M. Vaarkamp, I. Dring, R.J. Oldman, E.A. Stern and D.C. Koningsberger, Phys. Rev. B 50 (1994) 7872.

[2] P.H. Citrin, P. Eisenberger and B.M. Kincaid, Phys. Rev. Lett. 22 (1976) 3551.

[3] B.A. Bunker and E.A. Stern, Phys. Rev. B 27 (1983) 1017.

[4] B. Lengeler, J. Phys. (Paris) 47 (1986) 75.

[5] B.K. Teo and P.A. Lee, J. Am. Chem. Soc. 101 (1979) 2815 . 